\title{
Long-standing Intraspinal Glass Fragments Causing Subsequent Radiculopathy After Dorsal Stabilization -Case Report-
}

\author{
Florian Roser, Rainer RITZ, Florian H. EBNER, Dirk FREUDENSTEIN, \\ and Marcos TATAGIBA
}

Department of Neurosurgery, University of Tübingen, Tübingen, Germany

\begin{abstract}
A 37-year-old female presented with a history of lumbar intraspinal glass fragments due to an accident in childhood. The patient developed progressive right convexity thoracolumbar scoliosis during puberty. Twenty-eight years after the accident, horizontalization of this deformity was performed by dorsal stabilization. Postoperatively the patient complained of acute L-5 radiculopathy. Radiological examination detected multiple glass fragments intra- and extradurally around the L3-4 levels with compression of the dural sac. Microsurgical removal of the extra- and intradural glass fragments led to complete relief of the radicular pain. Foreign bodies can become symptomatic due to changes in the status of the spine, especially during growth in young patients.
\end{abstract}

Key words: intraspinal foreign body, stabilization, radiculopathy

\section{Introduction}

Progressive compressive radiculopathy due to foreign bodies in the spinal canal is well recognized. ${ }^{2,3,10)}$ Neurogenic claudication can occur many years after trauma as a result of retained foreign bodies within the spinal canal. Such retained fragments cause scar tissue and adhere to the neighboring structures. ${ }^{5)}$ Migration through the whole axis of the spine may occur. ${ }^{8,9,12)}$ Changes in the status of the vertebrae, due to stabilization or any other trauma, might alter the fragile stability of the spinal canal contents.

Here we describe a case of progressive thoracolumbar scoliosis caused by long-standing retention of glass fragments, which was treated by orthopedic ventrodorsal internal stabilization without laminectomy but resulted in induction of progressive radiculopathy requiring decompression surgery.

\section{Case Report}

A 37-year-old female presented with a history of lumbar back pain persisting for more than 20 years. She had accidentally fallen through a glass door at

Received August 29, 2005; Accepted May 9, 2006 the age of 9 years, when only superficial laceration at the lumbar spine was identified. She remained asymptomatic so no further investigation was performed. During puberty she developed progressive thoracolumbar scoliosis with complaints of lumbar pain and intermittent radiating pain during exercise in the bilateral ventral thighs.

Radiography of the thoracolumbar spine showed a right convexity scoliosis with $50^{\circ}$ Cobb angle and a clinically active left convexity scoliosis in the lumbosacral spine (Fig. 1). Subtle sclerotic changes were also detected immediately below the L-3 lamina. Computed tomography (CT) of the lumbar spine demonstrated foreign bodies which were apparently localized extradurally, so there was no contraindication for the planned orthopedic surgery.

Ventrodorsal internal stabilization without laminectomy was performed at the L3-5 levels in an outside orthopedic department to achieve horizontalization of the fused segments towards the sacrum (Fig. 2). The preoperative bending images suggested good spontaneous correction, but the thoracolumbar scoliosis remained uncorrected, so secondary fusion of T10-L3 was necessary. However, the orthopedic surgeon detected a substantial number of intraspinal glass fragments adhering to the dura, with scar tissue around the spinal dura. These fragments were left in situ to prevent damage to the intradural 

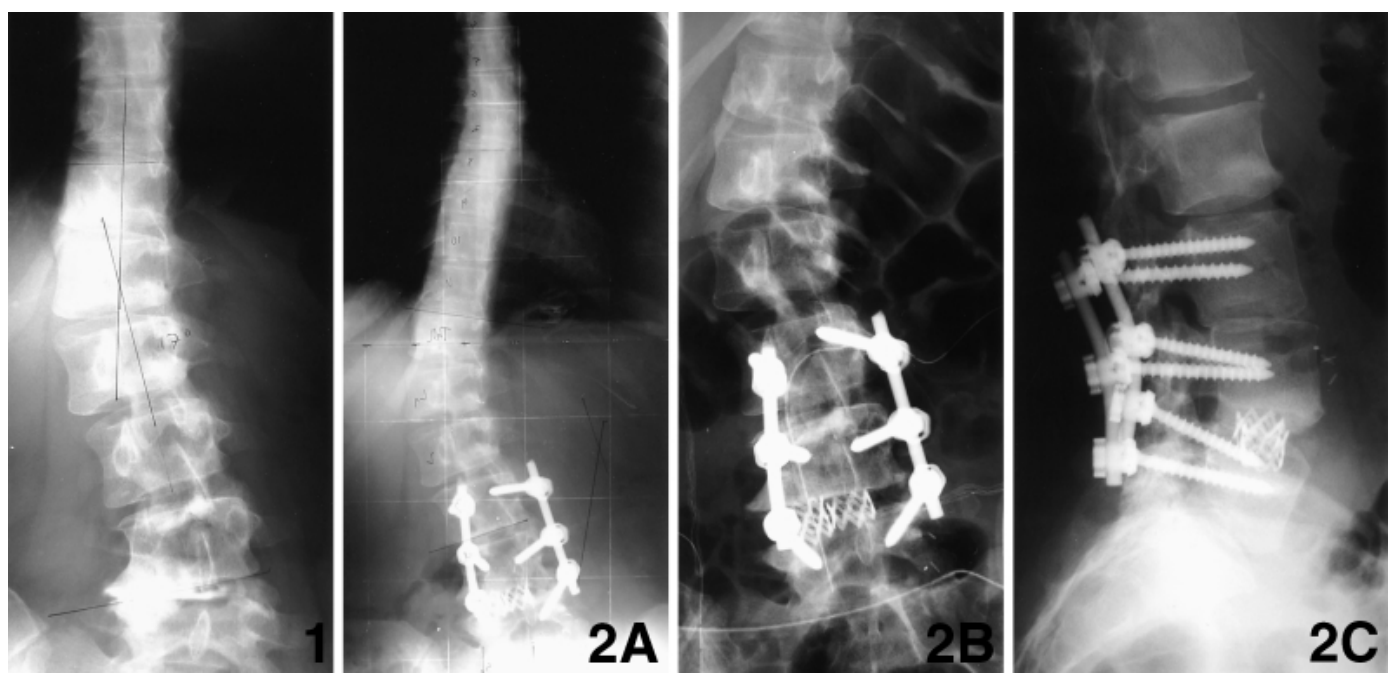

Fig. 1 Radiograph showing thoracolumbar scoliosis prior to orthopedic surgery, with sclerotic changes immediately below the L-3 lamina.

Fig. 2 Postoperative radiographs of the thoracolumbar spine, anteroposterior $(A, B)$ and lateral views (C), demonstrating the fused segments at the L3-5 levels.

structures. Whether the fragments were located extradurally, intradurally, or both remained unclear.

Postoperatively the patient was transferred to our neurosurgical department. Initial neurosurgical examination demonstrated L-5 compression syndrome with slight paresis of the right extensor hallucis muscle and hypesthesia over the L-5 dermatome. Lasègue sign was positive on the right, but pyramidal signs were absent. Bowel and bladder functions were intact. Postmyelography CT of the lumbar spine demonstrated significant compression of the thecal sac at the L3-4 levels caused by the foreign bodies with compression of the cauda equina (Fig. 3A, B). Surgery was planned for the new and progressive radiculopathy with neurological deficits.

Posterior spinal decompression of L3-4 was performed at our institution 1 month after the orthopedic operation. Multiple small glass fragments located paraspinally around L-4 were removed, followed by two large fragments penetrating the dural sac completely from the dorsal to the ventral aspects (Fig. 3C). Significant amounts of adhesive scar tissue had aggravated the compressive effect as the glass fragments were enclosed by granulomatous tissue. After removal of these fragments, the dura was closed in a watertight fashion.

Postoperatively the patient was fully mobilized from the 3rd postoperative day onwards. The radiculopathy resolved completely and the neurological deficits improved. No cerebrospinal fluid fistula
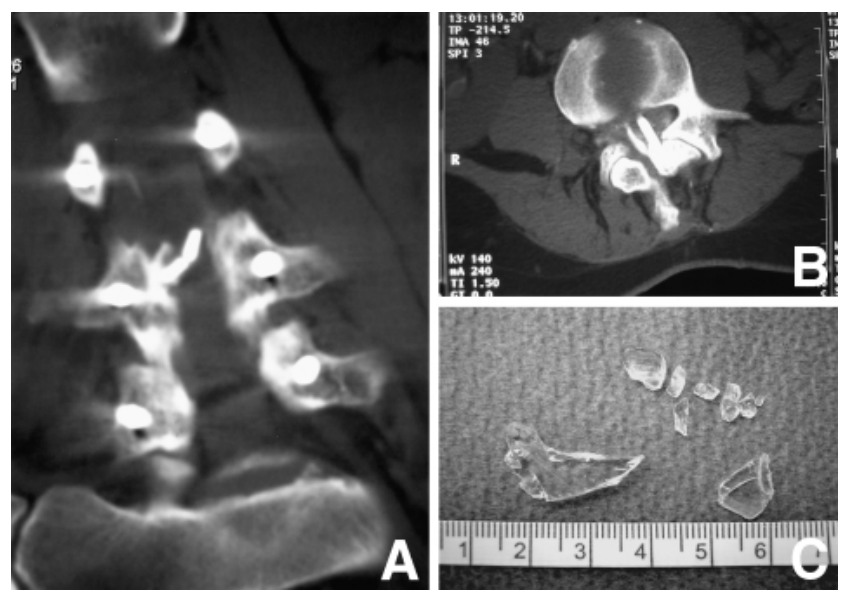

Fig. 3 A, B: Postmyelography computed tomography scans, coronal (A) and axial views (B), showing a foreign body nearly dividing the spinal canal in half (3-mm slice at the pedicular level of L-4). C: Photograph showing the removed glass fragments. The largest piece on the left corresponds to the fragment seen in (B).

was detected. One year postoperatively the patient was free of symptoms of nerve root compression.

\section{Discussion}

There are multiple potential etiologies for spinal deformities: Congenital scoliosis or kyphosis, neural 
tube defects, neuromuscular disorders, and any form of bone dysplasia. ${ }^{4,11)}$ None of these possible factors were present in the history of our patient. Obviously the traumatic intrusion of the foreign bodies within the spinal canal associated with longstanding back pain was the probable cause of the muscular imbalance with consecutive development of thoracolumbar scoliosis during adolescence. The erection of the thoracolumbar spine due to dorsal spondylosyndesis at L3-5 might have then facilitated the development of new neurogenic claudication due to disruption of the balanced nerve root-foreign body relationship. However, neurosurgeons should also consider that in the treatment of radiculopathy caused by degenerative lumbar spinal stenosis with scoliosis, the L-3 or L-4 roots are more strongly compressed by foraminal stenosis at the concave side of the curve, whereas the lower nerve roots are affected more by lateral recess stenosis at the convex side. ${ }^{7)}$ Therefore, the postoperative changes in the distribution of shearing powers at the dura and scar tissue around the foreign body may have led to further compression of the cauda fibers and consecutively to radiculopathy.

The presence or absence of neurological symptoms is generally considered to be the guide for further diagnostic procedures if a foreign body is retained in the vicinity of the spinal canal. ${ }^{1,10)}$ Only if neurological deficits occur, which might be after many years, surgical intervention should be considered, depending on the severity and type of the deficit as well as the age of the patient. ${ }^{6)}$ However, the present case suggests that intraspinal foreign bodies in younger patients need different surgical strategies. In particular, surgical removal of even 'silent' foreign bodies should be carefully considered in young patients to prevent the development of spinal deformities during growth.

\section{References}

1) Avci SB, Acikgoz B, Gundogdu S: Delayed neurological symptoms from the spontaneous migration of a bullet in the lumbosacral spinal canal. Case report.
Paraplegia 33: 541-542, 1995

2) Braunsdorf WE, Ellams ID: Spinal compression syndromes caused by intradural foreign body. Neurochirurgia (Stuttg) 30: 123-124, 1987

3) Ehni G: Occult foreign bodies in the spinal canal. Report of two cases. N Engl J Med 308: 947-949, 1983

4) Emans JB: Scoliosis and Kyphosis Stabilization, in Kaye AH, Black PM (eds): Operative Neurosurgery. London, Edinburgh, New York, Philadelphia, St Louis, Sydney, Toronto, Harcourt Publishers, 2000, pp 1995-2011

5) Fung CF, Ng TH: Delayed myelopathy after a stab wound with a retained intraspinal foreign body: case report. J Trauma 32: 539-541, 1992

6) Kuijlen JM, Herpers MJ, Beuls EA: Neurogenic claudication, a delayed complication of a retained bullet. Spine 22: 910-914, 1997

7) Liu $H$, Ishihara $H$, Kanamori $M$, Kawaguchi $Y$, Ohmori K, Kimura T: Characteristics of nerve root compression caused by degenerative lumbar spinal stenosis with scoliosis. Spine J 3: 524-529, 2003

8) Oge HK, Aydin S, Cagavi F, Benli K: Migration of pacemaker lead into the spinal venous plexus: case report with special reference to Batson's theory of spinal metastasis. Acta Neurochir (Wien) 143: 413-416, 2001

9) Satow T, Motoyama Y, Yamazoe N, Isaka F, Higuchi K, Nabeshima S: Migration of a lumboperitoneal shunt catheter into the spinal canal-case report. Neurol Med Chir (Tokyo) 41: 97-99, 2001

10) Tekavcic I, Smrkolj VA: The path of a wounding missile along the spinal canal: a case report. Spine 21: 639-641, 1996

11) Weinstein S: Idiopathic scoliosis: long-term followup and prognosis in untreated patients. J Bone Joint Surg Am 63: 702-712, 1981

12) Yasui K, Kotani Y, Takeda Y, Minami A: Migration of intracranial hemostatic clip into the lumbar spinal canal causing sacral radiculopathy: a case report. Spine 28: E511-E514, 2003

Address reprint requests to: F. Roser, M.D., Department of Neurosurgery, University of Tuebingen, HoppeSeyler-Str.3, 72076 Tuebingen, Germany.

e-mail: f.roser@gmx.de 